\title{
Ressources naturelles, développement et conflits : liens de causalité et mesures politiques
}

Natural Resources, Development and Conflict:
Channels of Causation and Policy Interventions

Paul Collier*

Oxford University et Banque Mondiale

De nombreux gouvernements des pays en développement perçoivent des recettes importantes grâce à la taxation des rentes issues de l'exploitation des ressources naturelles. Leurs conséquences en matière de développement ont souvent été négatives et paraissent très nettement en deçà de leur potentiel. Cet article s'intéresse aux moyens dont disposent les pays développés pour aider les gouvernements récipiendaires à faire un meilleur usage de leurs ressources. Dans un premier temps, sont considérés six canaux par lesquels les rentes tirées des ressources naturelles sont sources de problèmes : recherche de rente, encouragement à la sécession, financement des rebellions, «détachement » du gouvernement, syndrome hollandais et exposition aux chocs. Sont ensuite proposés quatre remèdes à ces problèmes : transparence des revenus, contrôle des dépenses, suivi des flux de produits et fourniture de mécanismes de protection contre les chocs.

Mots clés : ressources naturelles, gouvernance, conflit

Key words : natural resources, governance, conflict

Many governments of developing countries receive large revenues derived from taxation of natural resource rents. The developmental consequences of these revenues have often been negative and are clearly far below their potential. This paper focuses on how developed country governments could assist recipient governments to make better use of their own resources. It first considers six routes by which natural resource rents create problems : rent-seeking, encouraging secession, financing rebellion, the

* Les résultats, interprétations et conclusions exprimées dans cet article sont uniquement attribuables à l'auteur. Ils ne représentent pas nécessairement les points de vue de la Banque Mondiale, de ses Directeurs Exécutifs ou des pays représentés. 
detachment of government, Dutch disease, and exposure to shocks. It then proposes four remedies which address these problems - revenue transparency, the scrutiny of expenditures, the tracking of commodity flows, and the provision of cushions for shocks.

\section{INTRODUCTION}

Les ressources naturelles sont distribuées de manière aléatoire sur la surface du globe. Comme dans le cas de toute distribution aléatoire, le résultat est inégal. Certains pays ont des ressources naturelles en faibles quantités ou bien de faible valeur, alors que d'autres en ont en quantité. Cette abondance donne lieu à des opportunités d'accroissement des revenus. Historiquement, les dotations en charbon et en minerai de fer ont souvent eu un rôle crucial comme éléments initiateurs du développement. Depuis au moins les quatre dernières décennies, l'abondance de ressources a toutefois été fréquemment associée à des problèmes de potentiels de croissance non exploités et à de forts risques de conflits violents et étendus. Qu'une bonne fortune tourne au problème peut évidemment être évité et, récemment, la communauté internationale s'est demandée s'il existait des actions collectives réalisables permettant de réduire ces risques de manière significative. Pour dégager des interventions possibles, il est nécessaire de se pencher sur les raisons de ces effets néfastes de l'abondance de ressources naturelles. Bien sûr, la solution à tout problème ne nécessite pas obligatoirement que l'on s'adresse à ses causes; toutefois, sans une bonne compréhension de celles-ci, on peut douter de la pertinence des remèdes proposés.

\section{L'ASSOCIATION DE RESSOURCES NATURELLES ET DE FAIBLES REVENUS}

Le lien entre les ressources naturelles et le risque de guerre civile a été récemment exploré, à la fois au travers de l'analyse économétrique et d'études de cas. Les études de cas sont plus pertinentes pour la compréhension des mécanismes qui sont en jeu, tandis que l'économétrie est plus appropriée pour établir si l'on peut en tirer des relations de causalité.

La première étude économétrique ayant mis en évidence une relation entre les ressources naturelles et le risque de guerre civile était Collier 
et Hoeffler (1998). Dans la dernière version de notre travail (Collier et Hoeffler 2004a), nous analysons 54 conflits civils majeurs survenus entre 1965 et 1999. On observe alors qu'une plus grande part des exportations de matières premières dans le PIB accroît le risque de conflit de manière substantielle et significative. Par exemple, en comparant des pays dont les PIB proviennent à hauteur de $10 \%$ et $25 \%$ des ressources naturelles, toutes choses égales par ailleurs dans le cas de pays en développement à faibles revenus, le risque de guerre civile dans les cinq ans à venir passe de $11 \%$ à $29 \%$. En se demandant s'il existe des différences significatives entre les différents types de ressources, nous avons observé que le pétrole est la seule à se distinguer. Une forte dépendance au pétrole est associée à des risques plus importants de conflit civil qu'un niveau de dépendance aussi élevé pour les exportations d'autres produits primaires. Il faut toutefois rappeler que la dépendance aux ressources naturelles - ou, plus largement, la dépendance vis-à-vis des matières premières - ne constitue pas, de loin, le seul facteur jouant de manière significative dans le risque de conflit. En particulier, le niveau de revenus par habitant influence fortement le risque de conflit. Avec ou sans ressources naturelles, le risque de guerre civile est négligeable pour de hauts niveaux de revenus par habitant. Ainsi, des sociétés comme celles de la Norvège ou de l'Australie, pays qui dépendent fortement des exportations de ressources naturelles mais qui sont aussi riches, n'encourent aucun risque significatif lié à leurs dotations en ressources naturelles.

Plusieurs études ont étudié la relation existant entre ressources naturelles et durée des conflits, séparément du risque de son déclenchement. Fearon et Laitin (2003) observent que la dépendance aux ressources augmente la durée des guerres civiles. Collier, Hoeffler et Soderbom (2004) obtiennent des résultats similaires. Ce qui est intéressant, c'est qu'ils parviennent à séparer dépendance initiale et effets des variations des prix mondiaux des exportations de matières premières durant le conflit. L'avantage est qu'un prix mondial est presque entièrement orthogonal à un conflit individuel et peut facilement être observé, ce qui en fait une variable explicative particulièrement sûre. Sans surprise, nous remarquons que les effets de ces prix dépendent du niveau initial d'exportation de ces ressources. Pour une part relativement forte de $30 \%$ des exportations de matières premières dans le PIB en début de conflit, une hausse postérieure et permanente de $10 \%$ du prix international des exportations conduirait à un allongement de $12 \%$ de la durée du conflit.

La corrélation entre la dépendance vis-à-vis des ressources naturelles et de faibles performances en termes de croissance est aussi confirmée 
par l'analyse économétrique et les études de cas. La plus connue de ces études économétriques est celle de Sachs et Warner (2001) qui observent que les ressources naturelles réduisent la croissance. Il existe aussi de nombreuses séries d'études de cas. Gelb (1989) constitue une analyse particulièrement bonne des économies pétrolières.

\section{DE LA DÉPENDANCE AUX RESSOURCES NATURELLES AUX PROBLÈMES DE DÉVELOPPEMENT}

De par leur nature, ces études économétriques ne permettent habituellement pas d'expliquer pourquoi ces corrélations sont observées. Dans cette partie, je propose six mécanismes explicatifs, me reposant essentiellement sur des éléments de théorie et des expériences individuelles. Ross (2003) et Isham (2003) en offrent d'excellentes analyses.

\subsection{La recherche de rente}

Une explication potentielle évidente est que les ressources naturelles constituent une véritable mine d'or que les groupes d'intérêt se disputent. Étonnant, les preuves d'un tel mécanisme sont relativement minces. S'il s'agissait d'un facteur explicatif important, ce raisonnement devrait aussi s'appliquer à l'aide, ainsi que l'a précisément proposé un économiste des conflits, Grossman (1992). Certains gouvernements reçoivent des flux d'aide importants. Pour se les approprier, il pourrait être avantageux de prendre la tête du gouvernement. Lorsque l'aide est instrumentée et introduite dans le modèle de conflit de Collier et Hoeffler, celle-ci ne présente aucun effet direct significatif ${ }^{1}$. Il est toutefois possible que les ressources naturelles représentent une source plus évidente de rente que l'aide.

Certaines études de cas mettent en évidence l'importance de motifs purs de recherche de rente. Aux Fidji, une tentative violente de coup d'État a été initiée par un homme d'affaire qui était le représentant local d'une entreprise privée américaine à la recherche d'un contrat d'exploitation. La tentative eut lieu juste après que le contrat fut accordé à une autre compagnie. Au Sierra Leone, un accord de paix prévoyait la concession au chef du groupe rebelle RUF de la vice-présidence du pays,

1 L'aide réduit indirectement le risque de conflit par le biais de ses effets en termes de croissance. Voir Collier et Hoeffler (2004c). 
mais ce dernier refusa jusqu'à ce que soit incluse dans l'offre la présidence du conseil contrôlant les mines de diamants. Malgré les tentatives des chefs rebelles de donner à leurs actions respectives une interprétation différente, on peut difficilement conclure que la recherche de rente sur les ressources naturelles ne motivait en rien leurs actions.

Il est souvent difficile de distinguer ce qui répond à des objectifs de pure prédation et ce qui répond à des objectifs politiques. Quelquefois, les objectifs peuvent évidemment évoluer dans le temps. Par exemple, les FARC furent à l'origine un mouvement politique rural radical, mais correspondent sûrement maintenant davantage à une entreprise narcotique. Certainement qu'au fur et à mesure que les revenus de la drogue ont joué un rôle central dans les actions de l'organisation, les idéalistes motivés par l'idéologie devinrent de moins en moins enclins à la rejoindre, contrairement aux criminels attirés par l'argent et la violence. De la même manière, dans la région du delta du Nigéria, un mouvement initialement lié à la lutte contre l'injustice et la dégradation de l'environnement évolua rapidement en guerre de gangs entre villages pour le contrôle de droits de protection et le vol de rançons. En 2004, celui-ci atteint une taille suffisamment importante pour que ses dirigeants soient en position de négocier avec l'État nigérian.

\subsection{Rendre les mouvements sécessionnistes crédibles}

Une variante de l'hypothèse de la mine d'or et pour laquelle on dispose de preuves plus importantes est que l'abondance de ressources naturelles encourage de violentes sécessions. De même que la distribution des ressources naturelles entre pays n'est pas égale, certaines zones au sein des pays richement dotés sont mieux pourvues que d'autres. Les individus vivant à proximité des ressources naturelles ont un intérêt économique évident à en revendiquer la propriété au détriment de leurs compatriotes. Comme les ressources naturelles sont en général considérées comme une propriété publique plutôt que privée, la revendication d'une propriété commune locale équivaut à une revendication d'indépendance. Les nations sont souvent l'agglomération récente d'anciennes entités politiques distinctes dont le processus d'intégration a fréquemment été contesté. Ainsi, dans de nombreux cas, les ressources naturelles sont situées dans des régions dont un groupe politique - bien que souvent marginal - revendique déjà l'autonomie. La présence de ressources naturelles permet à de tels groupes d'ajouter un argument économique crédible à ce qui n'est sinon certainement en grande partie qu'un appel «romanesque ». L'augmentation (sans violence) du natio- 
nalisme écossais entre les élections de 1970 et 1974 illustre bien cette transformation. Lors de la première de ces élections, ainsi que toutes les précédentes, le Scottish National Party n'obtenait qu'une faible part des votes ainsi qu'un seul siège au parlement. Lors des deux élections de 1974, il obtint $30 \%$ des votes. L'origine de cette transformation fut sans doute la forte hausse du prix international du pétrole à la fin de 1973 résultant de la guerre du Kippour. Soudainement, le pétrole des côtes écossaises fut perçu comme précieux: le parti fit campagne sur le slogan «son pétrole à l'Ecosse ». Bien sûr, les spéculations de l'époque ont sans doute accru la valeur qu'on lui prêtait bien au delà de sa vraie valeur. L'OPEP semblait pouvoir augmenter le prix sans limite, et le Shah d'Iran commençait à parler du pétrole comme du «noble combustible », donnant ainsi aux gisements pétroliers une aura de corne d'abondance. Un mouvement similaire de sécession, toujours lié au pétrole mais violent cette fois, eut lieu au Nigéria dans le Biafra. L'entrée du Biafra dans une violente sécession eut lieu peu après des mesures fiscales visant à traiter les revenus du pétrole comme un bien national. Même s'il existe des tensions ethniques au Nigéria, presque tous les pays africains ont plusieurs groupes ethniques. Il est tout de même frappant que les groupes ethniques qui ont tenté une sécession en Afrique étaient généralement riches en ressources naturelles, comme cela fut le cas au Biafra et au Katanga.

Habituellement, les groupes ethniques de régions pauvrement dotées ne recherchent guère la sécession, pour la bonne raison qu'ils seraient perdants. Lorsque des sécessionnistes romanesques parviennent néanmoins à établir une demande de sécession politiquement crédible sans doute du fait du charisme hors norme du leader - les autres régions peuvent décider d'accorder sans violence cette indépendance en l'absence d'opposition violente. Ce fut le cas en Tchécoslovaquie, où la pauvre région de Slovaquie obtint pacifiquement la sécession de la plus vaste et plus riche région tchèque.

Dans Collier et Hoeffler (2004b), nous avons réalisé une étude statistique des sécessions. Celle-ci repose sur la classification des guerres civiles en sciences politiques entre sécessionnistes versus idéologiques. Cette classification n'est pas irréprochable puisque dépendant dans une certaine mesure des objectifs apparents du groupe rebelle. Toutefois, à partir de cette distinction, nous avons testé si les exportations de matières premières avaient un effet différent dans les deux cas sur les risques de guerre civile. Nous avons observé que la dépendance aux matières premières augmentait davantage le risque de conflit sécessionniste que de guerre idéologique. Nous nous sommes par la suite intéressés spé- 
cifiquement au cas du pétrole et avons trouvé que lorsque qu'un pays exportateur de pétrole connaissait une guerre civile, celle-ci était quasiment systématiquement de nature sécessionniste, contrairement aux pays non exportateurs de pétrole qui ont fréquemment connu des conflits civils de nature idéologique.

Le pétrole peut se distinguer par sa connotation romanesque de prospérité. Par exemple, le GAM, mouvement rebelle qui tenta la sécession de l'Aceh vis-à-vis de l'Indonésie, instrumenta les similarités de la région avec le Brunei pour sa propagande, prétendant que la population de l'Aceh pouvait atteindre le même niveau de richesse. Il s'agit d'une exagération grossière et sans doute délibérée, mais elle flatta l'imagination de la population.

\subsection{Financement des groupes rebelles}

Un autre canal explicatif, par lequel les matières premières en général et les ressources naturelles en particulier peuvent accroître le risque de conflit civil, passe par leur vulnérabilité à la prédation par des groupes rebelles en mal de financement pour poursuivre leurs actions violentes. En général, la rébellion est coûteuse. Les recrues travaillent en général à temps plein et doivent ainsi être logées, vêtues et nourries. Plus particulièrement, l'armement coûte cher et tend à s'user rapidement lors des combats entre les mains de combattants mal entraînés. Beaucoup de prétendus groupes rebelles ne peuvent tout simplement pas financer leurs activités au delà d'actes mineurs de terrorisme. La prédation des ressources naturelles ne constitue en aucun cas le seul moyen de financement d'une armée rebelle, mais, lorsque celles-ci sont abondantes en zones rurales, elles sont particulièrement vulnérables car difficiles à défendre, immobiles et rentables. L'extorsion d'une rente sur l'exploitation des ressources naturelles des zones rurales nécessite aussi des techniques de violence très similaires à celles auxquelles recourent les groupes rebelles dans leur combat. On est à l'opposé de l'extorsion en milieu urbain où de nombreux types d'armements au delà des armes de poing constitueraient davantage un handicap qu'un atout.

Les exemples les plus remarquables de financement des groupes rebelles par des ressources naturelles sont liés aux diamants. L'UNITA et le RUF sont tous deux devenus des organisations de taille particulièrement importante - il fut un temps, on estimait que L'UNITA rassemblait 150000 hommes. De telles armées sont extrêmement coûteuses et reposent donc sur une prédation des ressources tout aussi importante. Les diamants alluviaux conviennent parfaitement à la prédation, car le 
processus d'exploitation est suffisamment simple pour ne pas nécessiter la présence de grandes entreprises. Des milliers de petits agents économiques sont dans une position bien moins confortable que de grandes entreprises pour se défendre, ce qui permet facilement aux organisations rebelles d'intimider et de pratiquer une taxation informelle sur les producteurs. Le bois convient aussi parfaitement à la prédation pour les rebelles d'un point de vue technologique, comme c'est le cas au Cambodge avec les Khmers rouges. Lorsque l'exploitation nécessite de grandes entreprises, comme pour le pétrole, les groupes rebelles peuvent toujours pratiquer la prédation, mais celle-ci change alors de nature. Les entreprises sont alors menacées de sabotage des pipelines, et leurs employés capturés et échangés contre rançon. Toutefois, il devient de plus en plus difficile pour les firmes multinationales de payer les groupes rebelles.

Un phénomène apparenté concerne les « options sur butin de guerre » (Ross, 2003). Dans ce cas, le groupe rebelle a besoin d'une grande entreprise à qui vendre, conditionnellement à une victoire de sa part, les perspectives hautement risquées de droits d'exploitation.

\subsection{Détachement du gouvernement vis-à-vis de son électorat}

Historiquement, des gouvernements représentatifs sont apparus car les États avaient besoin de prélever de larges sommes afin de mener leurs guerres et parce qu'ils se sont aperçus que l'acceptation des taxes par la population nécessitait en retour la concession d'une part du pouvoir aux contribuables. Un argument commun de politique économique, appliqué aussi bien aux ressources naturelles qu'à l'aide, est qu'en diminuant le besoin de taxation, celles-ci conduisent à une baisse de l'attention portée par la population sur le gouvernement. Les individus se sentent moins concernés par le mauvais usage de l'argent public s'ils n'ont pas été taxés pour financer celui-ci. La puissance de cet argument dépend du degré de sophistication de l'électorat. En principe, le coût d'opportunité d'un mauvais usage des fonds publics est le même quelle que soit leur source. Toutefois, le gouvernement qui fait mauvais usage de ces fonds peut plus facilement masquer l'étendue des revenus issus des ressources naturelles que ceux obtenus par le biais de la taxation. Il peut ainsi être facile de coopter le relativement petit groupe de personnes informées et potentiellement critiques vis-à-vis du mauvais usage des ressources naturelles.

Une variante de cet argument de «détachement» est que les rentes issues des ressources naturelles fournissent au gouvernement 
une autre option d'assise politique. Mes recherches actuelles avec Hoeffler concluent que les rentes issues des ressources naturelles pervertissent systématiquement le fonctionnement de la démocratie. Alors qu'en l'absence de ressources naturelles les démocraties surpassent les autocraties, le fonctionnement démocratique se détériore tellement en présence de rentes importantes que les autocraties leur prennent le pas. De fortes rentes issues des ressources naturelles permettent sans doute de maintenir le pouvoir par le biais d'appuis privés, plutôt que par les biens publics qu'une démocratie les forcerait à produire.

Dans la mesure où le gouvernement est d'autant plus détaché de considérations électorales qu'il tire ses recettes des ressources naturelles, ce phénomène est à la fois un mal en lui-même et une source potentielle de rébellion, sécession comprise. La perception d'un accaparement des ressources par une élite corrompue est toujours commode pour les groupes rebelles. En pratique, on retrouve rarement une seule motivation à la rébellion, et la perception de la corruption du gouvernement peut être un facteur de conflit, même s'il ne peut, seul, contribuer significativement au déclenchement de la violence.

\subsection{Syndrome hollandais}

Trente ans auparavant, la communauté économique universitaire fit grand cas du problème du syndrome hollandais - la tendance pour les exportations de ressources naturelles à apprécier le taux de change réel et à rendre ainsi la production des autres produits échangeables moins compétitive. La conséquence de ces exportations correspond simplement à une réallocation efficace des ressources - celles-ci passent dans le secteur des non-échangeables, permettant de répondre à l'accroissement de la demande lié à l'enrichissement de la société mais qui ne peut être satisfait par le biais des importations. Pour que l'on observe ce syndrome, on a fréquemment recours à au moins une des deux conditions suivantes. La première est que l'appréciation réelle est temporaire alors que parallèlement les acteurs privés ont une mauvaise perception de l'évolution intertemporelle des prix relatifs qui en découle. En conséquence, les facteurs de production sont entraînés dans le secteur des biens non échangeables où ils restent piégés lorsque l'évolution des prix se renverse. Ceci implique des coûts d'ajustement très importants. Deuxièmement, on peut supposer que les externalités comme le learning by doing sont plus importantes dans le secteur des biens échangeables non primaires, ce qui explique que la croissance soit moins forte. 
La même critique est quelquefois adressée à l'aide, celle-ci exerçant les mêmes effets d'appréciation réelle du taux de change. Les effets de l'aide sur la croissance ont longuement été débattus. Collier et Dollar (2002) utilisent une approche en forme réduite en contrôlant pour l'influence de la politique afin de retirer l'effet de « détachement » que l'aide et les ressources naturelles peuvent avoir en commun. On observe que l'aide présente des rendements décroissants et conditionnés à la politique. Le point à partir duquel l'effet de l'aide devient négatif est appelé « point de saturation ». Lorsque les politiques sont de qualité moyenne, l'aide contribue positivement à la croissance jusqu'à un niveau de 20 à $30 \%$ du PIB. Lorsqu'elles sont mauvaises, le point de saturation est observé pour des niveaux d'aide d'environ 10 à $15 \%$ du PIB. Comme les revenus des ressources naturelles sont relativement analogues à l'aide, du moins pour un environnement politique donné, on peut s'attendre à ce que ces estimations de capacité d'absorption s'appliquent autant à l'aide qu'aux revenus issus des ressources naturelles. La décroissance des rendements qui mène l'économie à son point de saturation, au delà duquel l'aide - et par extension les ressources naturelles - réduit la croissance, peut refléter le syndrome hollandais. Autrement dit, ces ressources peuvent avoir un effet bénéfique sujet au processus habituel de décroissance des rendements, et un effet adverse - le syndrome hollandais - qui devient éventuellement prépondérant. De nombreux pays richement dotés en ressources naturelles sont susceptibles de passer le «point de saturation», étant dans la zone où les recettes tirées des ressources naturelles exercent un effet marginal net négatif. Ceci s'explique en partie parce que ces revenus sont quelquefois très élevés - dépassant $30 \%$ voire $40 \%$ du PIB, comme c'est le cas au Nigéria. Toutefois, la qualité des politiques est aussi souvent faible, du fait peutêtre de l'effet de « détachement », ce qui ramène le point de saturation à des niveaux de revenus relativement bas. D'un point de vue dynamique, ceci peut amener un cercle vicieux. Avec de mauvaises politiques et des revenus issus des ressources naturelles élevés, la part de l'économie non liée aux ressources naturelles est susceptible de connaitre une croissance lente voire négative, donc de diminuer relativement à la composante liée à l'exploitation des ressources naturelles. Il est alors difficile de soutenir des réformes. Ce fut par exemple le sort du Nigéria où l'économie non pétrolière a quasiment stagné sur une longue période. Ainsi, savoir si le syndrome hollandais justifie une quelconque attention semble dépendre à la fois de l'environnement politique et de l'importance des revenus tirés des ressources. 


\subsection{L'exposition aux chocs}

Un dernier mécanisme par lequel les ressources naturelles, et évidemment d'autres matières premières, peuvent retarder le développement est lié à l'exposition de l'économie à des chocs de prix. De larges chocs négatifs tendent à donner lieu à des épisodes de contraction importante de l'économie qui s'ajoutent aux pertes directes de revenus. De tels épisodes augmentent directement la pauvreté du fait de la baisse des recettes d'exportation. Ils tendent aussi à réduire le taux de croissance de la production à moyen terme (Collier et Dehn, 2001). Au delà des seules pertes de revenus et de production, Hoeffler et moi-même avons observé que des épisodes de déclin rapide de l'économie augmentent substantiellement le risque de guerre civile. Même les chocs positifs peuvent déstabiliser la gestion de l'économie, privant celle-ci de certaines opportunités. Ce sujet est approfondi ailleurs, notamment dans la communication présentée à la Conférence ABCDE-Europe en même temps que celle dont est issue cet article par Patrick Guillaumont et alii (2003) ainsi que dans Guillaumont et Guillaumont Jeanneney (2003).

\section{LES RÉPONSES POLITIQUES À CES PROBLÈMES AU NIVEAU INTERNATIONAL}

Les six mécanismes passés en revue dans la section 3 et au travers desquels les ressources naturelles peuvent influer négativement sur le développement peuvent ne pas jouer simultanément dans un contexte particulier. Ils sont toutefois susceptibles de s'appliquer dans suffisamment de contextes pour justifier un intérêt international. Cependant, ils sont tous différents les uns des autres. Évidemment, aucune intervention politique ne peut répondre seule à ces six problèmes de manière simultanée. De fait, si tous ces mécanismes sont crédibles, comme cela semble être le cas, ce qui sera nécessaire est un ensemble d'interventions résolvant de concert tous ces problèmes. En l'absence d'un tel ensemble, des mesures s'adressant à un unique problème risquent de présenter des effets limités. Si l'un de ces mécanismes adverses ne joue plus, ceci peut accroître le risque de voir les autres prendre effet.

Je m'intéresse maintenant aux composantes d'un ensemble possible de mesures. Elles sont détaillées de manière plus ample dans Collier et alii (2003) et Bannon et Collier (2003). Bien que l'on ait six canaux, certaines mesures jouent sur plus d'un, ce qui me permet de penser que quatre éléments seulement suffisent pour disposer d'un ensemble efficace. 
Il s'agit de : la transparence des revenus; la surveillance des dépenses; le suivi des produits; et une faible exposition aux chocs de prix. Je vais maintenant me pencher alternativement sur les trois premiers, le dernier étant traité dans les études précitées de Guillaumont et alii.

\subsection{Transparence des recettes}

La transparence des revenus est utile sous de multiples aspects. Premièrement, elle est évidemment un élément nécessaire au contrôle des dépenses - si l'on ne connaît pas les recettes, il est vain de s'interroger sur l'utilisation qui en est faite. Un corollaire est que le secret autour des recettes va anéantir les efforts de surveillance des dépenses. Par rapport aux six mécanismes mis en évidence précédemment, la transparence est nécessaire pour résoudre le problème de «détachement». De plus, elle peut permettre de réduire le problème de pression sécessionniste. Rappelons, à partir de l'exemple du GAM dans l'Aceh, que le mouvement rebelle essayait délibérément d'exagérer la valeur des revenus tirés des ressources naturelles. Plutôt que de laisser la population dans l'indifférence, le secret est susceptible de faciliter de telles exagérations. La transparence seule ne suffit toutefois pas à contrer ce type de propagande car de nombreux gouvernements de pays en développement n'ont pas la confiance de leur population en matière d'information, en particulier pour des sujets aussi contestés. La riposte d'un groupe rebelle comme le GAM à la campagne d'information d'un gouvernement, montrant des recettes pétrolières inférieures à celles présentées par le groupe, est simplement d'accuser le gouvernement de détournement. Ainsi, tout système de transparence doit également être crédible pour la population locale.

L'ONG Global Witness a mené des efforts pour encourager la transparence, militant pour une évaluation obligatoire des entreprises d'exploitation des ressources. Bien que la proposition présente certains avantages, elle a aussi ses inconvénients. Premièrement, il est difficile d'un point de vue politique de réaliser une action internationale sur une base obligatoire: certaines firmes verraient là une violation de la confidentialité, et les gouvernements percevant des revenus issus des ressources naturelles la recevraient comme une nouvelle insinuation de corruption de la part des pays développés. Deuxièmement, si les entreprises émettaient leurs rapports pour des périodes d'exercice comptable différentes, ou avec des concepts différents de recettes, il serait impossible de dégager des statistiques crédibles au niveau agrégé : des rapports individuels seuls pourraient paradoxalement, en fournissant 
une information trop désagrégée, donner trop peu d'information pour assurer une surveillance efficace. Cette approche comprend aussi le risque d'oubli du rôle clef des compagnies pétrolières nationales. Une approche alternative, suggérée par le gouvernement britannique, consiste à ce que les entreprises soient sollicitées par le gouvernement du pays riche en ressources naturelles pour rendre des comptes de manière confidentielle à la Banque Mondiale qui publierait par la suite des informations au niveau agrégé. Cette méthode a plusieurs avantages. Premièrement, le gouvernement aurait le choix d'exiger ou non cette procédure. Ainsi il ne serait plus lui-même le sujet d'une obligation, mais deviendrait un acteur primordial dans la production de transparence. À partir du moment où un «modèle » aura été établi pour rendre compte des recettes, les gouvernements qui demanderaient aux firmes de rendre des comptes conformément à ce modèle signaleraient leur engagement sur la voie d'une bonne gouvernance. Cette possibilité de signal serait elle-même la plus utile pour les gouvernements ayant à faire face à un problème de mauvaise réputation - cela offre un mécanisme par lequel ils peuvent faire oublier leur passé. Deuxièmement, le gouvernement exigerait des comptes de toutes les entreprises, y compris des entreprises nationales. Ceci permettrait d'éviter que, si les exigences de comptes provenaient des pays de l'OCDE, celles-ci ne s'appliqueraient qu'à certaines entreprises et les désavantageraient donc - ce qui rend les pays de l'OCDE peu enclins à imposer des obligations aussi discriminatoires. Troisièmement, en introduisant un intermédiaire qualifié, comme la Banque Mondiale, il est possible d'obtenir que les données présentées reprennent certains concepts standards. Ceci peut réduire les problèmes de rapports pouvant induire en erreur, et peut aussi faciliter une agrégation satisfaisante. L'intermédiaire peut par la suite communiquer les données agrégées sous une forme qui soit suffisamment peu technique pour respecter l'esprit comme la lettre de la transparence. Finalement, comme le gouvernement du pays exportateur de ressources naturelles provoquerait le processus, il serait naturel que les rapports établis par les entreprises soient accompagnés d'informations du gouvernement sur les recettes perçues, de manière à ce que les versements déclarés soient en accord avec les recettes déclarées.

\subsection{Surveillance}

La transparence des recettes est elle-même un élément du contrôle des dépenses. Sans transparence des revenus, l'examen des dépenses perdrait beaucoup de son efficacité, mais la transparence n'est pas en elle-même 
de la surveillance. Celle-ci est essentiellement un processus national: ceci parce que les citoyens et leurs représentants sont les bénéficiaires légitimes des recettes publiques. La communauté des donneurs d'aide peut aussi avoir un intérêt légitime dans le contrôle, dans la mesure où elle injecte des ressources dans le pays, mais sa place est clairement secondaire par rapport aux intérêts nationaux. Si la surveillance reposait uniquement sur les récriminations des gouvernements étrangers et des agences internationales, il serait facile pour les gouvernements interpellés de se protéger en recourant à la stratégie maintes fois éprouvée du nationalisme.

L'objectif du contrôle est d'établir comment les recettes tirées des ressources naturelles sont dépensées. Comme les revenus sont fongibles, ceci peut uniquement être fait au travers d'un examen minutieux de tout le processus budgétaire. Dans la plupart des pays en développement richement dotés en ressources naturelles, les institutions qui devraient normalement prendre en charge cette surveillance - des commissions parlementaires, associées à un organisme général de contrôle des comptes et une presse d'investigation - ne sont en général pas suffisamment efficaces. L'acquisition de capacités de contrôle repose en partie sur la formation, et en partie sur un changement du gouvernement vers l'acceptation voire l'encouragement des recherches. À nouveau, le gain d'un tel changement de comportement est plus important pour les gouvernements handicapés par une mauvaise réputation - aussi bien auprès de leur propre électorat que des investisseurs étrangers.

Dans certains cas, les institutions de contrôle doivent être établies ex nihilo. Par exemple, le gouvernement du Tchad a mis en place, du fait de la construction d'un oléoduc entre le Tchad et le Cameroun, de nouvelles institutions avec la participation d'un groupe ad hoc incluant la société civile et les entreprises étrangères. Jusqu'à aujourd'hui, l'expérience semble suggérer que même cette approche ad hoc a été relativement efficace. La construction précise du contrôle va obligatoirement différer d'un pays à l'autre, dépendant de ce qui est actuellement en place.

Ensemble, transparence et contrôle devraient permettre de répondre à trois des six mécanismes par lesquels les ressources naturelles sont à la source des problèmes de développement. Premièrement, ils devraient réduire les conflits autour des «mines d'or» en diminuant la capacité de détourner les ressources pour des besoins illégitimes. Deuxièmement, ils permettraient de réduire les incitations à la sécession. Les groupes sécessionnistes ne pourraient plus exagérer la taille des revenus, pas plus qu'ils ne pourraient faire de lien entre perspectives d'accumulation de revenus au niveau local et détournements au niveau 
national. Une perception crédible que les recettes sont employées pour des dépenses équitables d'un point de vue national, comme l'éducation primaire, constitue la meilleure défense contre la sécession. Enfin, transparence et contrôle permettent de répondre un minimum au problème de «détachement ». Progressivement, la population est amenée à considérer les recettes tirées des ressources naturelles comme appartenant à la nation, la manière dont elles sont utilisées devenant ainsi une préoccupation majeure en termes de politique intérieure.

\subsection{Suivi des produits}

Le suivi des produits a été récemment expérimenté au travers du plan Kimberley de suivi des diamants. Celui-ci est encore récent et il est nécessaire d'établir son degré d'efficacité. Toutefois, s'il s'avère qu'il fonctionne, il pourrait être étendu à d'autres produits comme le bois et le pétrole. Le principal objectif du suivi des produits est que les groupes rebelles ne puissent profiter que plus difficilement du pillage des ressources naturelles précieuses. Comme certains groupes importants, comme l'UNITA, le RUF et les Khmers Rouges en ont fait leur principale source de revenus, la mesure peut avoir un impact particulièrement important sur les finances des groupes rebelles. Elle ne consiste pas réellement à écarter du marché les ressources pillées par les rebelles, mais plutôt à les contraindre à être vendues à très bas prix. La bonne estimation de l'efficacité du suivi des produits est donc simplement dans l'observation des prix bas que les rebelles sont obligés d'offrir. Même si l'information n'est pas publique, celle-ci est relativement facilement accessible auprès des personnes informées de l'industrie. Ce prix est ainsi beaucoup plus aisé à suivre que les quantités actuelles d'un produit prohibé mises sur le marché.

Le suivi physique des produits peut aussi être utilement associé avec des informations sur les transactions financières qui représentent la contrepartie de leurs mouvements physiques. Une telle intégration des systèmes d'expertise financière, qui sont à la charge des banques, et des expéditions physiques, qui sont contrôlées par les institutions douanières, serait relativement simple avec des technologies modernes d'information, et augmenterait considérablement l'information sur la transparence. Actuellement, les obligations de contrôle des banques sont grandement accrues pour lutter contre la corruption et le terrorisme, de même que les obligations de contrôle pour les mouvements physiques, mais les deux n'ont pas été associées. 
Ces stratégies de suivi répondent en priorité au problème que les ressources naturelles ont historiquement été utilisées pour le financement des mouvements rebelles. En augmentant la transparence, elles répondent aussi aux questions de sécession et de « détachement »- on voit le gouvernement tenter de réduire l'utilisation illicite des recettes.

\subsection{Exposition moindre aux chocs}

Cet élément de l'ensemble de mesures est traité plus en profondeur dans les études de Guillaumont et alii (2003). Les stratégies de réduction de l'exposition aux chocs de prix consistent, dans les grandes lignes, en une protection via des assurances publiques ou privées, une diversification des exportations, et, de manière plus ambitieuse, une réduction des chocs de prix eux-mêmes.

L'assurance privée peut être réalisée au travers d'une couverture sur les marchés internationaux des produits. Les gouvernements ont rarement tenté une telle couverture et il s'agit elle-même d'une procédure risquée. Un élément particulièrement désincitatif est que la couverture est politiquement dangereuse - cette stratégie présente approximativement $50 \%$ de chance de se concrétiser par des pertes financières, au moins dans le court terme, et, dans ce cas de figure, les opposants critiqueront les preneurs de décision. Ceci est risqué car la réalisation de contrats sur les marchés dérivés constitue une forme complexe de pari, et, si les responsables publics sont autorisés à s'y engager, ils seront quelquefois tentés de prendre des risques plutôt que de les réduire. La surveillance de ces responsables est difficile et coûteuse. Par contre, il est possible pour la Banque Mondiale de prendre en charge la gestion de ces contrats de couverture - n'entrant pas ellemême sur le marché, mais agissant au nom des gouvernements clients, et supportant les risques de la surveillance des équipes responsables. La Banque Mondiale gère déjà les positions de change de quelques pays en développement. La gestion de couvertures pour les matières premières ne semble donc poser aucune question de principe.

Il existe plusieurs options en matière d'assurance publique. Le FMI ou la Banque Mondiale pourraient introduire des facilités de prêt conditionnel, initiées par de graves chutes des prix des exportations. La communauté des donneurs pourrait fournir l'aide en réponse à des chocs de prix de la même manière qu'elle le fait actuellement pour des chocs plus médiatiques comme pour des tremblements de terre ou des inondations. Finalement, la Banque est un créancier important à la fois pour des pays exportateurs de matières premières et des pays 
importateurs de matières premières. La plus importante étant ici le pétrole. Actuellement, le service de la dette des pays exportateurs de pétrole est d'environ 6 milliards de dollars par an, et celui des pays importateurs de pétrole est d'à peu près 12 milliards de dollars par an. De fait, il existe au moins en principe une possibilité pour ces deux flux d'aller dans le sens d'une compensation, conditionnée par le prix du pétrole. Lorsque le prix du pétrole est élevé, les exportateurs de pétrole prendraient en charge une partie du service de la dette des importateurs de pétrole, et vice et versa. La Banque y gagnerait en réduisant ses risques par défaut, et les importateurs comme les exportateurs de pétrole par des revenus nets moins volatils.

La diversification et la diminution de la volatilité des prix elle-même pourraient être soutenues par un changement de politique commerciale des pays de l'OCDE. Actuellement, l'escalade des tarifs par les pays de l'OCDE réduit la rentabilité du traitement des matières premières dans les pays en développement par rapport à l'extraction des ressources. Accorder des subventions agricoles contingentes au prix mondial a pour effet d'accroître les mouvements de prix. Par exemple, lorsque le prix mondial du coton a diminué, le gouvernement américain a augmenté les subventions à ses producteurs de coton. Ceci a accru davantage la baisse des prix internationaux pour les producteurs de coton de la République Centrafricaine.

De telles stratégies répondent directement au problème de la volatilité des prix. Toutefois, associées à la transparence et au contrôle, elles ont aussi pour effet de réduire le problème du syndrome hollandais. Rappelons que le syndrome hollandais est mieux appréhendé en termes de capacité d'absorption - quel niveau de recettes issues des ressources naturelles le gouvernement peut-il utiliser de manière productive? La capacité d'absorption dépend à son tour de la qualité de l'environnement politique. En réduisant les fluctuations économiques et en augmentant l'efficacité de la dépense publique, la capacité d'absorption est accrue, ce qui permet que moins de pays aient à se trouver dans des situations où l'appréciation réelle du taux de change devient la principale considération.

\section{CONCLUSION}

Les agences de développement se concentrent actuellement sur «l'efficacité de l'aide» et la «cohérence politique». L'efficacité de l'aide correspond au besoin d'augmenter les effets de l'aide sur la croissance 
et la réduction de la pauvreté; la cohérence politique correspond aux tentatives d'accorder des politiques apparemment disparates comme l'aide et le commerce, de les rendre toutes capables d'appuyer le développement. Les revenus tirés des ressources naturelles revenant aux pays en développement sont bien plus importants que les flux d'aide, mais ils sont analytiquement identiques. La cohérence politique implique que la communauté internationale s'intéresse à la hausse des rendements des revenus tirés des ressources naturelles, tout comme elle s'est démenée pour augmenter les rendements de l'aide. Évidemment, la contrepartie à la hausse des rendements des revenus issus des ressources naturelles est une réduction de ceux de l'aide. Certaines des actions requises sont similaires - comme le contrôle national -, d'autres sont totalement différentes. Mais les longs débats relatifs à l'efficacité de l'aide contrastent avec la négligence qui a prévalu jusqu'à une période récente en matière de politique internationale relative aux ressources naturelles. 


\section{RÉFÉRENCES}

BAnnon, I. et P. Collier (eds) (2003), Natural Resources and Violent Conflict: Actions and Options, World Bank.

Collier, P., L. Elliott, H. Hegre, A. Hoeffler, M. Reynal-Querol et N. SAmBanis (2003), Breaking the Conflict Trap: Civil War and Development Policy, Oxford University Press for World Bank.

Collier, P. et D. Dollar (2002), "Aid Allocation and Poverty Reduction", European Economic Review, 46, pp. 1475-1500.

Collier, P. et J. DeHn (2001), “Aid, Shocks and Growth", World Bank Policy Research Working Paper 2688.

Collier, P. et A. Hoeffler (1998), "On the Economic Causes of Civil War", Oxford Economic Papers, 50, pp. 563-73.

Collier, P. et A. Hoeffler (2004a), "Greed and Grievance in Civil War", Oxford Economic Papers, 56, pp. 563-595.

Collier, P. et A. Hoeffler (2004b), "The Political Economy of Secession", in H. Hannum et E.F. Babbitt (eds.) Negotiating Self-Determination.

Collier, P. et A. Hoeffler (2004c), "Violent Conflict", in Bjorn Lomborg (ed.), Global Problems, Global Solutions, Cambridge University Press.

Collier, P., A. Hoeffler et M. Soderbom, (2004), "On the Duration of Civil War", Journal of Peace Research.

FEARON, J. et D. LAitin (2003), "Ethnicity, Insurgency and Civil War", American Political Science Review, 97(1).

GelB, A. et associés (1989), Oil Windfalls:Blessing or Curse?, Oxford University Press.

Grossman, H. (1992), "Foreign Aid and Insurrection", Defence Economics, 3, pp. $275-288$.

Guillaumont, P., S. Guillaumont Jeanneney (2003), "Dampening Price Shocks", in I. Bannon et P. Collier (eds.).

Guillaumont, P., S. Guillaumont Jeanneney, P. Jacquet, L. Chauvet et B. SAVOYE (2005), "Attenuating through Aid the Vulnerability to Price Shocks", à paraître in ABCDE Conference 2003 Proceedings, Oxford University Press.

Isham, J., L. Pritchett, M. WoolCOCK et G. Busby (2003), "The Varieties of the Resource Experience: How Natural Resource Export Structures Affect the Political Economy of Economic Growth", mimeo, World Bank, Washington DC.

Ross, M. (2003) "The Natural Resource Curse : How Wealth Can Make You Poor", in Bannon et Collier (eds.).

SACHS, J. et A. WARNER (2001), "The Curse of Natural Resources", European Economic Review, 45, pp. 827-38. 